\title{
Manuscripts Using Numerical Discrete Element Methods
}

\author{
Giovanni Barla $\cdot$ Herbert Einstein • \\ Kalman Kovari
}

Published online: 31 May 2013

(c) Springer-Verlag Wien 2013

The creation of numerical discrete element methods was a breakthrough in modeling discontinuous media and thus in modeling of rock masses. Recent developments in this domain, as also shown in a Special Issue of our Journal (Volume 45, Issue 5, September 2012), make it possible to simulate rock on any scale from intact rock composed of several particles to rock masses.

The simultaneous increase in computer power greatly facilitates the use of these numerical models. As a consequence, much research and many presentations in papers use these models. Somewhat unfortunately, much of the work represents only incremental differences from what was done earlier or in parallel by the same researchers or by others.

Particularly problematic is the fact that modeling rarely goes hand in hand with physical investigations in the laboratory or in the field. Otherwise expressed, running a test with a numerical model is much faster, cheaper and can involve more parameters than the corresponding physical test. Responsible researchers realize that simulation always requires comparison with physical reality.
Similarly, when numerical discrete element methods are used in engineering practice and design to examine complex problems and study the influence of various parameters on the rock mass response, the need arises to ensure that the calculations performed by such methods lead to realistic results which can be checked and well understood.

The editors recognize that our ability to simulate rock and rock masses has been greatly advanced through these tools. We welcome papers that compare simulations with laboratory experiments or field observations, and we also welcome papers that make a significant contribution to the numerical modeling itself.

On the other hand, we have to be restrictive if manuscripts simply present marginally modified applications of numerical models. Such manuscripts will be rejected without peer review. We are aware that our readers and authors understand that in doing this we will maintain the quality of our journal, something that we all desire.

\footnotetext{
G. Barla $(\bowtie)$

Politecnico di Torino, Torino, Italy

e-mail: giovanni.barla@polito.it

H. Einstein

Massachusetts Institute of Technology, Cambridge, USA

K. Kovari

Oberengstringen, Switzerland
} 